\title{
ORIGINAL RESEARCH \\ Local Thrombolysis for Severe Cerebral Venous Sinus Thrombosis
}

X.-b. Guo
S. Guan
Y. Fan
L.-j. Song

C erebral venous and sinus thrombosis accounts for $0.5 \%-1 \%$ of all strokes and is one of the common causes of stroke in the young. ${ }^{1}$ The standard therapy for cerebral venous and sinus thrombosis is anticoagulation with intravenous unfractionated heparin or subcutaneous low-molecular-weight heparin. ${ }^{2}$ Even though there has been a steady decline, recent reports still indicate a mortality of $5 \%-30 \%{ }^{3}$ The predictors of poor outcome include coma, ICH, rapidly progressing clinical deficits, posterior fossa lesions, and involvement of the deep venous system. Approximately $30 \%$ of patients with 1 or more of these risk factors had poor outcome despite treatment with heparin. ${ }^{4}$ For these patients, endovascular thrombolysis might give better results. Failure to respond to adequate systemic anticoagulation has been considered as a possible indication of local intrasinus thrombolysis. Evidence from small case series suggests that local thrombolytic therapy is relatively safe and effective in rapid recanalization of thrombosed sinus and reversal of neurologic deficits. ${ }^{4,5}$

Local intrasinus thrombolysis dissolves the thrombus by infusion of a thrombolytic drug into the occluded sinus. The

Received July 17, 2011; accepted after revision September 20.

From the Departments of Interventional Radiology (X.-b.G., S.G.) and Neurosurgery (L.-j.S.), The First Affiliated Hospital of Zhengzhou University, Zhengzhou, China; and Department of Neurosurgery (Y.F.), Tianjin Huanhu Hospital, Tianjin, China.

Please address correspondence to Lai-jun Song, Department of Neurosurgery, The First Affiliated Hospital of Zhengzhou University, 1 Jianshe Rd, Zhengzhou, China, 450052; e-mail: aijunsong@sina.com

Indicates open access to non-subscribers at www.ajnr.org

http://dx.doi.org/10.3174/ajnr.A2932 theoretic advantage of intrasinus thrombolysis for cerebral venous and sinus thrombosis is that the drug is delivered where needed. However, in the absence of randomized controlled trials, the exact role of intrasinus thrombolysis in the management of cerebral venous and sinus thrombosis is unclear, especially with regard to patient selection, optimal time to intervene, and contraindications to this therapy. We treated 37 patients with severe cerebral venous and sinus thrombosis with intrasinus thrombolysis, analyzed the results, and compared them with results of previous studies.

\section{Materials and Methods}

Thirty-seven patients with dural venous sinus thrombosis were retrospectively identified at 2 institutions (Tianjin Medical University, China, and University of Zhengzhou, China) from January 2007 to November 2009. The patients included in the study were treated with local intrasinus thrombolysis. Anticoagulation with conventional heparin and warfarin was the initial treatment in all patients. The anticoagulation treatment consisted of heparin $1 \mathrm{U} / \mathrm{kg}$, intravenous once every 12 hours, and warfarin dosage adjusted to maintain an INR of 2-3. Both heparin and warfarin were given in every patient. PTT monitoring was performed to adjust the dose of heparin. Patients were offered intrasinus thrombolysis when there was 1) lack of adequate clinical response to heparin, 2) rapid worsening of neurologic deficits, or 3) an assumed poor prognosis because of an altered mental status (cognitive disturbances including abnormal alertness and orientation, coma), straight sinus thrombosis, or large space occupying stroke. The diagnosis of cerebral venous and sinus thrombosis was confirmed by CT, MR imaging, and MR venography, or by conventional angiography. Informed consent for intrasinus throm- 


\begin{tabular}{|c|c|}
\hline & Number of Patients \\
\hline \multicolumn{2}{|l|}{ Patient characteristics } \\
\hline Male & 12 \\
\hline Mean age (year and range) & $31(17-42)$ \\
\hline Onset to diagnosis (days and range) & $3(1-8)$ \\
\hline Diagnosis to thrombolysis (days and range) & $1.5(0.5-2)$ \\
\hline GCS (range) & $8.4(5-13)$ \\
\hline \multicolumn{2}{|l|}{ Clinical presentation } \\
\hline Headache & $34(92 \%)$ \\
\hline Visual disturbances & $7(20 \%)$ \\
\hline Aphasia & $9(24 \%)$ \\
\hline Seizures & $16(43 \%)$ \\
\hline Sensory symptoms & $2(5 \%)$ \\
\hline Mental status disorder & $7(20 \%)$ \\
\hline Hemiparesis & $19(51 \%)$ \\
\hline Coma & $5(14 \%)$ \\
\hline Intracranial hemorrhage or infarct & $23(62 \%)$ \\
\hline
\end{tabular}

Note:- Total number of patients $=37$.

bolysis was taken from the patient or a relative of the patient after explaining the risks and benefits of the procedure. The pretreatment CT or MR imaging scans were assessed for midline shift, hemorrhage, and lesion size.

The thrombolytic treatment technique consisted of the advancement of a 6 F guiding catheter to the jugular bulb or sigmoid sinus over the guidewire. A microcatheter was then advanced via the internal jugular vein and into a frontal position in the superior sagittal sinus or straight sinus. The catheter was left in situ, and continuous urokinase $(42,000 \mathrm{U} / \mathrm{h}$, total $1,000,000 \mathrm{U} /$ day $)$ was infused into the superior sagittal sinus or superior sagittal sinus + straight sinus in each patient. The catheter was pulled out $2-3 \mathrm{~cm}$ every day and check angiograms were performed each day. The infusion was discontinued after potency of the superior sagittal sinus was identified on the repeated angiogram or in the presence of clinical deterioration. Heparin was continued during thrombolytic therapy. The doses of heparin were adjusted to maintain a PTT between 50 and 60 seconds. MRV was performed to assess the outcome 2 weeks after thrombolysis. All patients in the thrombolysis group were subsequently started on longterm oral anticoagulation (warfarin therapy).

Sinus recanalization was examined by contrast injection through the thrombolysis catheter or by MRV. Patients were clinically followed-up for 6 months and the outcome was measured using MRS, with the following scale: 0 , normal; 1 , no significant disability; 2 , slight disability (look after own affairs without assistance); 3 , moderate disability (need help, able to walk when assisted); 4, moderately severe disability (unable to walk unassisted); 5, severe disability (unable to ambulate, altered mentation); 6, death. Treatment response was assessed as good improvement (MRS 0, 1, 2), partial improvement (MRS 3, 4), or poor outcome (MRS 5, 6).

\section{Results}

\section{Baseline Characteristics}

We treated 37 patients ( 25 women) with a mean age of 31 years (range 17-42 years). The mean GCS was 8.4 (range 5 to 13 ). The median number of days between onset of symptoms and diagnosis was 3 (range 1-8 days). The median number of days between diagnosis to thrombolysis was 1.5 (range 0.5 to 2). Seven patients were comatose before treatment. Sixteen patients had tonic-clonic seizures, with an epileptic status in 2.

\section{Table 2: Causes or risk factors}

\begin{tabular}{lc}
\hline Cause or Risk Factors & Number of Patients (\%) \\
\hline Oral contraconceptives & $7(19 \%)$ \\
Hormonal manipulation & $3(8 \%)$ \\
Pregnancy or puerperium & $11(30 \%)$ \\
Patients with malignancies & $1(3 \%)$ \\
High estrogen levels (male) & $3(8 \%)$ \\
Nephrotic syndrome & $2(5 \%)$ \\
Infective causes & $6(16 \%)$ \\
Dehydration & $1(3 \%)$ \\
No risk factor identified & $3(8 \%)$
\end{tabular}

Table 3: Dural sinus involvement in patients with cerebral venous sinus thrombosis

\begin{tabular}{lc}
\hline Sinus Involved & Number of Patients (\%) \\
\hline SSS alone & $7(19 \%)$ \\
SSS + Unilateral TS & $15(41 \%)$ \\
SSS + Bilateral TS & $11(30 \%)$ \\
SSS + StrS & $4(10 \%)$
\end{tabular}

Note:-SSS indicates superior sagittal sinus; StrS, straight sinus; TS, transverse sinus

Other presenting clinical features are given in Table 1. Risk factors for cerebral venous and sinus thrombosis are shown in Table 2. The superior sagittal sinus (alone or in combination with other venous sinuses) was the most commonly affected sinus. The sinuses affected are listed in Table 3.

\section{Treatment Characteristics}

The technical success rate was $100 \%$. Thirty-three patients (superior sagittal sinus and superior sagittal sinus + transverse sinus) in the thrombolysis group received local thrombolysis into the superior sagittal sinus. The mean time of urokinase treatment was 6.2 days (range 5 to 7 ). Improvement of the superior sagittal sinus was noted on MRV in $32 / 33$ (97\%) patients (Fig 1). Complete recanalization of the superior sagittal sinus was seen in $27 / 33(82 \%)$ patients and partial recanalization was found in $5 / 33(15 \%)$ patients. No recanalization of the unilateral transverse sinus was found in $12 \mathrm{pa}-$ tients. Of the 33 patients (superior sagittal sinus and superior sagittal sinus + transverse sinus) at discharge, 25 patients recovered or had minimal residual symptoms (MRS of 0 or 1 ), 6 patients had a minor handicap (MRS of 2), 1 patient had moderately severe disability (MRS of 4), and 1 died from severe edema in the basal ganglia and thalamic areas, increased amount of parenchymal blood as seen on the CT scan, and transtentorial herniation.

Four patients (superior sagittal sinus + straight sinus) in the thrombolysis group were comatose before treatment and received local thrombolysis into the superior sagittal sinus and straight sinus with 2 microcatheters at the same time. The mean time of urokinase treatment was 5.5 days (range 5 to 6). Complete recanalization of the superior sagittal sinus was seen in $3 / 4(75 \%)$ patients (Fig 2). One patient deteriorated while undergoing thrombolysis because the patient was in a poor clinical condition, with rapidly worsening clinical symptoms, eventually becoming unresponsive and entering a vegetative state and deep coma. Thrombolysis was performed as a last therapeutic option, with very little hope for recovery, and MR imaging showed the hemorrhagic infarct enlarged during 

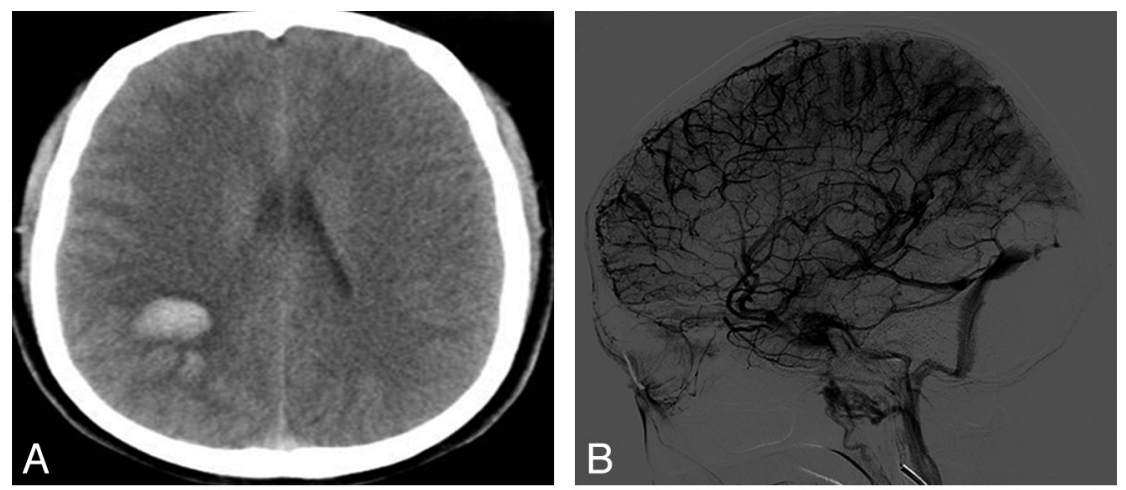

Fig 1. A 32-year-old woman presented with acute onset severe headache, vomiting, and left hemiparesis. A, CT scan revealed intracranial hemorrhage and infarct. $B$, DSA revealed features of cerebral venous sinus thrombosis involving superior sagittal sinus, left transverse sinus. $C$, Microcatheter was advanced into the superior sagittal sinus. $D, M R V$ showed complete recanalization of superior sagittal sinus after 6-day local urokinase administration. The patient was asymptomatic at the time of discharge.
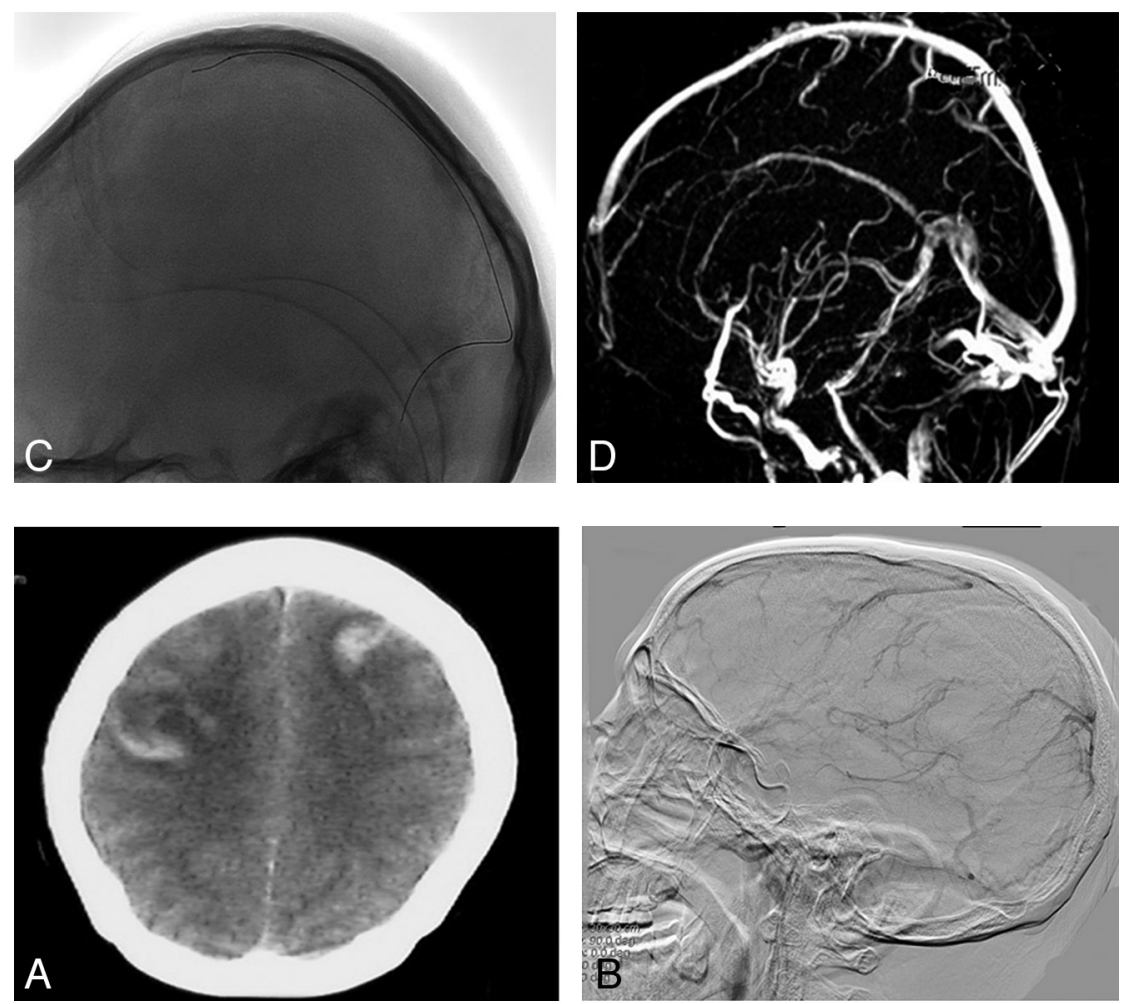

Fig 2. A 36-year-old woman, who took oral contraconceptives for several years, presented with acute onset severe headache, vomiting, and generalized seizure, and slipped into coma. $A$, CT scan revealed intracranial hemorrhage and infarct. $B$, DSA revealed features of extensive cerebral venous sinus thrombosis involving superior sagittal sinus, straight sinus, transverse sinus, and deep venous system. $C$, One microcatheter was advanced into the superior sagittal sinus, and another microcatheter was advanced into the straight sinus (arrows show the mark of microcatheter). $D$, MRV showed complete recanalization of superior sagittal sinus, straight sinus, transverse sinus, and deep venous system after 7-day local urokinase administration. The patient was asymptomatic at the time of discharge.
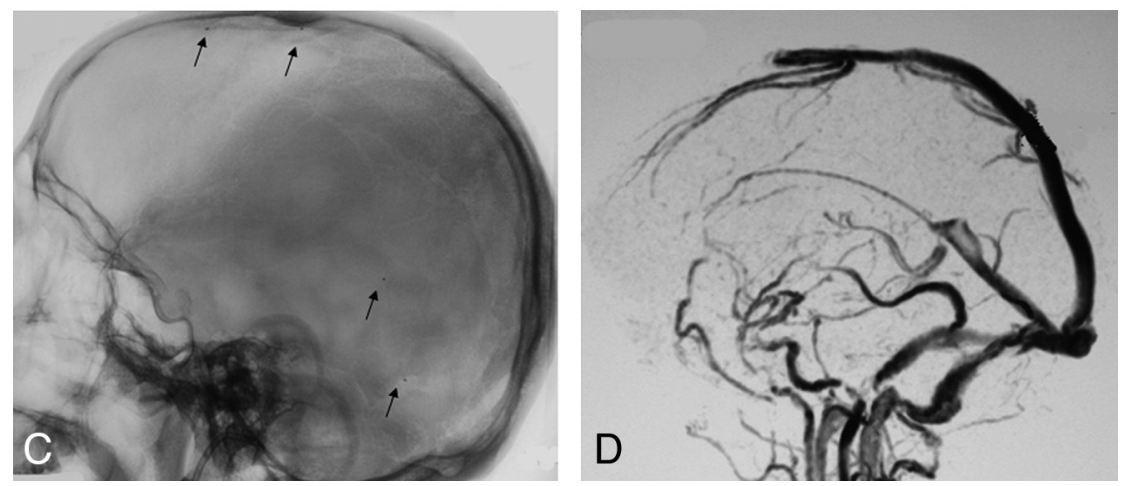

thrombolysis treatment. Of the 4 patients (superior sagittal sinus + straight sinus) at discharge, 2 recovered and had few residual symptoms (MRS of 1 ), 1 patient came out with a minor handicap (MRS of 2), and 1 patient died.

\section{Long-Term Follow-Up}

The 6-month follow-up rate was high, with data available for all discharge patients. All patients received oral anticoagulants for at least 6 months after discharge. Patients with normal thrombophilia profiles at 6 months did not receive further anticoagulation. At the last clinical follow-up, 34 patients $(92 \%)$ were either asymptomatic or had only minor subjective symptoms.

\section{Discussion}

Use of local pharmacologic thrombolysis in cerebral venous and sinus thrombosis was first reported in 1988 by Scott et al. They infused urokinase directly into the superior sagittal sinus 
via a midline frontal burr-hole. The patient, at first decerebrate, improved rapidly over several days. ${ }^{6}$ Subsequently, several authors have published case reports and small case series highlighting the usefulness of intrasinus thrombolysis in cerebral venous and sinus thrombosis. The largest case series included 20 patients with intrasinus thrombolysis. ${ }^{7-10}$ In our study, local urokinase was relatively well tolerated in 35 patients. Recanalization was documented in 35 patients receiving urokinase. Ninety-two percent of patients had excellent recovery or minor handicaps. One died, related to transtentorial herniation, an increased amount of parenchymal blood associated with thrombolysis, and 1 patient died because she was in a poor clinical condition before thrombolysis, with very little hope for recovery. Thus, death appears to be an uncommon direct complication of thrombolysis in cerebral venous and sinus thrombosis.

Intracranial hemorrhage is a feared complication of thrombolytic therapy. In our study, 23 patients in our thrombolysis group had pretreatment hemorrhagic infarct. Worsening of these hemorrhages or progressive neurologic deficits were identified in 2 of these patients. Chow et al described 2 patients treated with rheolytic thrombectomy and direct urokinase thrombolysis. ${ }^{11}$ Both patients had pretreatment intracranial hemorrhages, and 1 exhibited worsening of a preexisting hemorrhage and developed a new hemorrhagic infarction. Both of these patients had excellent recovery. Kim and Suh described 9 patients, 1 of whom had pretreatment hemorrhage that remained stable. ${ }^{12}$ Kumar et al described 19 patients, and 2 of these patients showed fatal worsening of hemorrhages after thrombolysis. ${ }^{13}$

There is currently no evidence from randomized controlled trials about the efficacy and safety of either systemic or local thrombolytic therapy in patients with cerebral venous and sinus thrombosis. Thrombolytic therapy has the potency to provide faster restoration of venous outflow, and positive effects of local thrombolytic treatment of cerebral venous and sinus thrombosis have increasingly been reported in uncontrolled series. ${ }^{14,15}$ Intravenous thrombolysis has resulted in variable outcomes. It can take days for high-dose peripheral infusion of streptokinase and urokinase to recanalize vessels, as the concentration of the thrombolytic agent that actually arrives at the site of occlusion is low. Thrombolysis is accelerated through direct intrasinus infusion of the thrombolytic agent. ${ }^{16,17}$ There is also a lower incidence of hemorrhage associated with locally catheter-administered thrombolytic agents. In this study, recanalization was documented in 35 patients receiving urokinase. There was also an incidence of hemorrhage associated with local thrombolytic agents related to 2 patients.

There are no established criteria in the literature regarding the indications for thrombolysis in cerebral venous and sinus thrombosis. ${ }^{18}$ Buccino and colleagues suggested the following criteria: risk of death, involvement of deep venous system, and poor clinical response despite full heparinization, ${ }^{19}$ whereas Stam and colleagues considered intrasinus thrombolysis in patients with altered mental status, coma, straight sinus thrombosis, or large space-occupying lesions. ${ }^{20}$ In our study, according to our inclusion criteria, rapid and sustained recovery was observed in $92 \%$ patients. Rapid resolution of neurologic dys- function, particularly in patients unresponsive to intravenous anticoagulation with heparin, indicates the importance of infusing an adequate concentration of fibrinolytic agent at the site of thrombosis. Early therapeutic intervention may lead to successful clot lysis.

\section{Conclusions}

Cerebral venous sinus thrombosis is an increasingly recognized cause of neurologic illness, with a range of clinical symptoms. This study highlights the benefit of thrombolysis, particularly in patients unresponsive to anticoagulation. Small case series have demonstrated safety and good initial results with direct thrombolytic infusions. The major limitation of our study is that it is an observational study and not a randomized study, and the patients who received intrasinus thrombolysis and who did not receive intrasinus thrombolysis were not comparable. Well-designed randomized controlled trials comparing heparin infusion and intrasinus thrombolysis alone, or in combination with heparin, are very much required.

\section{References}

1. Bousser MG, Ferro JM. Cerebral venous thrombosis: an update. Lancet Neurol 2007;6:162-70

2. Einhäupl K, Bousser MG, de Bruijn SF, et al. EFNS guideline on the treatment of cerebral venous and sinus thrombosis in adult patients. Eur J Neurol 2010; 17:1229-35

3. Ferro JM, Canhão P, Stam J, et al. Prognosis of cerebral vein and dural sinus thrombosis: results of the International Study on Cerebral Vein and Dural Sinus Thrombosis (ISCVT). Stroke 2004:35:664-70

4. Rahman M, Velat GJ, Hoh BL, et al. Direct thrombolysis for cerebral venous sinus thrombosis. Neurosurg 2009;27:E7

5. Wasay M, Bakshi R, Kojan S, et al. Nonrandomized comparison of local urokinase thrombolysis versus systemic heparin anticoagulation for superior sagittal sinus thrombosis. Stroke 2001;32:2310-17

6. Scott JA, Pascuzzi RM, Hall PV, et al. Treatment of dural sinus thrombosis with local urokinase infusion, case report. J Neurosurg 1988;68:284-7

7. Peng Y, Xuan JG, Yang YL, et al. Endovascular thrombolysis for severe cerebral sinus thrombosis. Interv Neuroradiol 2009;15:407-12

8. Hsu FP, Kuether T, Nesbit G, et al. Dural sinus thrombosis endovascular therapy. Crit Care Clin 1999;15:743-53

9. Newman CB, Pakbaz RS, Nguyen AD, et al. Endovascular treatment of extensive cerebral sinus thrombosis. J Neurosurg 2009;110:442-45

10. Sujith OK, Krishnan R, Asraf V, et al. Local thrombolysis in patients with dural venous thrombosis unresponsive to heparin. J Stroke Cerebrovasc Dis 2008;17: 95-100

11. Chow K, Gobin P, Saver J, et al. Endovascular treatment of dural sinus thrombosis with rheolytic thrombectomy and intra-arterial thrombolysis. Stroke 2000;31:1420-25

12. Kim Y, Suh JH. Direct endovascular thrombolytic therapy for dural sinus thrombosis: infusion of altepase. AJNR Am J Neuroradiol 1997;18:639-45

13. Kumar S, Rajshekher G, Reddy CR, et al. Intrasinus thrombolysis in cerebral venous sinus thrombosis: single-center experience in 19 patients. Neurol India 2010;58:225-29

14. Ferro JM, Canhão P. Acute treatment of cerebral venous and dural sinus thrombosis. Curr Treat Options Neurol 2008;10:126-37

15. Canha P, Falca F, Ferro JM. Thrombolytics for cerebral sinus thrombosis. A systematic review. Cerebrovasc Dis 2003;15:159-66

16. Hocker SE, Dafer RM, Hacein-Bey L. Successful delayed thrombolysis for cerebral venous and dural sinus thrombosis: a case report and review of the literature. J Stroke Cerebrovasc Dis 2008;17:429-32

17. Tsai FY, Kostanian V, Rivera M, et al. Cerebral venous congestion as indication for thrombolytic treatment. Cardiovasc Intervent Radiol 2007;30:675-87

18. Yamini B, Loch Macdonald R, Rosenblum J. Treatment of deep cerebral venous thrombosis by local infusion of tissue plasminogen activator. Surg Neurol 2001;55:340-46

19. Buccino G, Scoditti U, Pini M, et al. Loco-regional thrombolysis in the treatment of cerebral venous and sinus thrombosis: report of two cases. Acta Neurol Scand 2001;103:59-63

20. Stam J, Majoie CB, van Delden OM, et al. Endovascular thrombectomy and thrombolysis for severe cerebral sinus thrombosis: a prospective study. Stroke 2008;39:1487-90 